\title{
Recurrent primary mediastinal liposarcoma: A case report
}

\author{
CHUN ZHAO, FANGBIAO ZHANG, XIANGYAN ZHANG, SHAOSONG TU, ZHIJUN WU, \\ XIA LI, YINGMING XIANG, CHUNHUI ZHENG and QINGHUI ZENG
}

Department of Cardiothoracic Surgery, Lishui Center Hospital, Lishui, Zhejiang 323000, P.R. China

Received December 25, 2014; Accepted March 1, 2016

DOI: $10.3892 / 01.2016 .4453$

\begin{abstract}
Primary mediastinal liposarcomas are extremely rare. The current study reports the case of a 63-year-old man presenting with a primary liposarcoma arising from the posterior mediastinum. The patient reported a 6-month history of chest pain with increasing dyspnea for 2 months. Enhanced computed tomography revealed a $10 \times 16-\mathrm{cm}$ mass in the posterior mediastinum. Other physical examinations were normal. Radical resection was performed under the agreement of patient. Subsequent pathological analysis indicated a liposarcoma. The patient recovered and was successfully discharged. However, at a follow-up examination 12 months after surgery, recurrence was identified in the anterior mediastinum. Therefore, the patient underwent surgery. The postoperative course was uneventful, however, there was evidence of disease recurrence 2 years after the second surgery. The patient refused any treatment and succumbed after 3 months.
\end{abstract}

\section{Introduction}

Liposarcomas originate from primitive mesenchymal cells (1). According to the World Health Organization, they are divided into five subtypes: Dedifferentiated, pleomorphic, myxoid, round cell and well-differentiated (2). Primary liposarcomas arising from the mediastinum are uncommon and constitute $<1 \%$ of all mediastinal tumors (3). According to statistics reported by Kashu et al (3), the most common sites of primary mediastinal liposarcomas are the lower extremities (75\%) and the retroperitoneum (20\%). Mediastinal liposarcomas are insidiously growing tumors, which extend into the pleural spaces or compress the contiguous structures, including the esophagus, lung, superior vena cava and pericardium (1). Due to the rarity of this tumor type, there is currently no standard treatment regime. Complete resection alone or in combination with chemotherapy or

Correspondence to: Mr. Xiangyan Zhang, Department of Cardiothoracic Surgery, Lishui Center Hospital, 289 Kuocang Road, Lishui, Zhejiang 323000, P.R. China

E-mail: zhangxiangyan9@126.com

Key words: mediastinal liposarcoma, surgery, recurrent radiotherapy has been performed by a number of surgeons (4). In addition, mortality and survival rates of mediastinal liposarcoma remain uncertain, since cases of this disease are rare. The current study describes a case of recurrent primary liposarcoma in the mediastinum of a 63-year-old man, with histopathological and clinical analysis, and review of previously reported cases in the literature.

\section{Case report}

A 63-year old man was referred to Lishui Center Hospital (Lishui, China) in July 2005 with a 6-month history of chest pain and increasing dysphagia for 2 month. The patient had a history of cigarette smoking, but no history of diabetes mellitus, hypertensive disease or coronary disease. A chest computed tomography (CT) scan (Brilliance iCT; Philips Healthcare, Amsterdam, The Netherlands) revealed a large mixed solid mass involving the posterior mediastinum and expanding to the left pleural cavity (Fig. 1). Other physical examinations, including electrocardiogram, lung functional examination and transesophogeal echocardiogram, were normal. Distant metastasis was not found by head magnetic resonance imaging (MRI) and abdominal ultrasound. However, as the possibility of malignancy could not be ruled out, a left posterolateral thoracotomy was performed in the fourth intercostal space under general anesthesia on August 3, 2005. During thoracotomy, it was observed that the tumor tissue was multilobulated and the pseudocapsule was intact. There was less invasion of the tumor into the left lung and close adhesion of aortic arch, esophagus diaphragm, pericardium or left principal bronchus. The tumor was resected intact, weighed 2,464 g (Fig. 2) and measured 24x22x16 cm in size. Resected tumor tissue was formalin-fixed, paraffin-embedded and cut into $4-\mu \mathrm{m}$ sections. Histopathological examination using hematoxylin and eosin staining (Sinopharm Chemical Reagent Co., Ltd., Shanghai, China) revealed the tumor to have common histological findings of liposarcomas with scattered atypical and hyperchromatic stromal cells (Fig. 3) (3). The patient was discharged on the tenth postoperative day after an uneventful course. The patient was followed up 6 months later.

The patient again presented to Lishui Center Hospital for dysphagia 1 year later in July 2006. A preoperative chest CT scan revealed a massive anterior mediastinal tumor expanding to the left pleural cavities (Fig. 4). The tumor was consider to be recurrent liposarcoma, thus, resective surgery was proposed. A left anterolateral thoracotomy was performed 


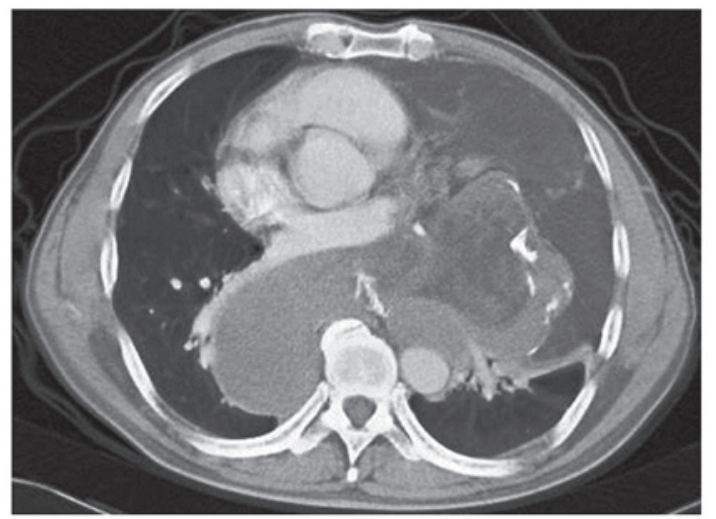

Figure 1. Chest computed tomography indicating a large mass in the posterior mediastinum and left cavity.

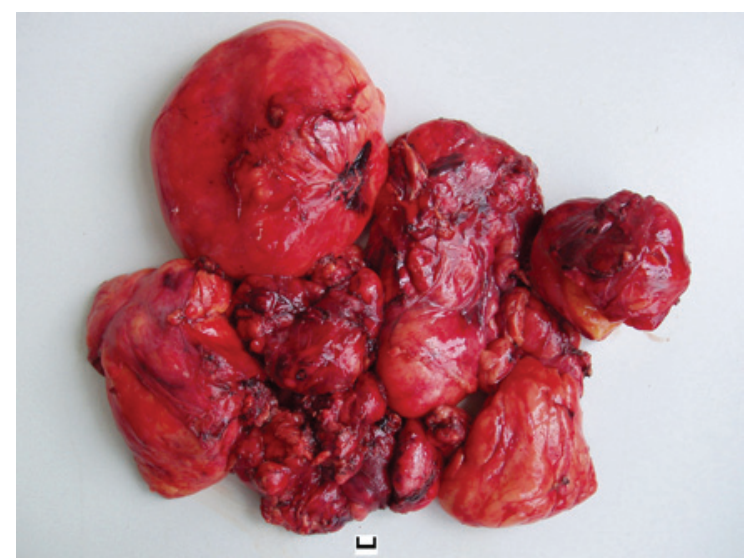

Figure 2. Macroscopic view of the tumor. The tumor tissue was multilobulated and the pseudocapsule was intact. Scale bar, $1 \mathrm{~cm}$.

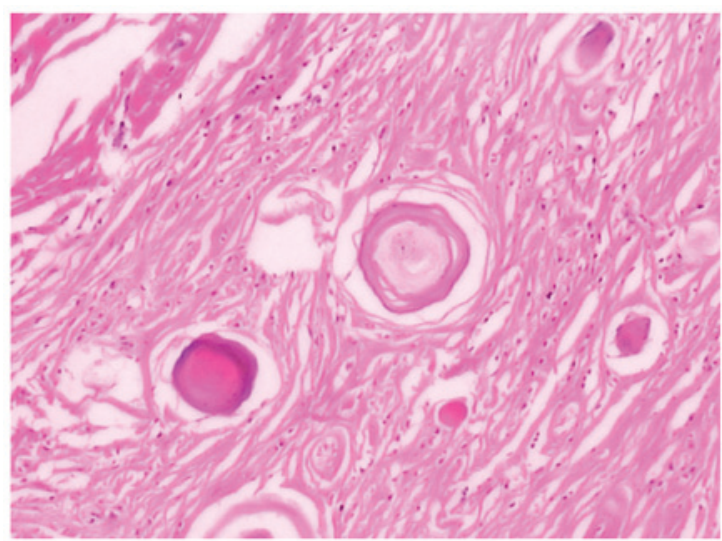

Figure 3. Pathological examination revealing scattered atypical and hyperchromatic stromal cells (hematoxylin and eosin staining; magnification, $\mathrm{x} 100)$.

in the fourth intercostal space and the tumor was complete resection. The massive tumor weighed $980 \mathrm{~g}$ with dimensions of 16x12x8 cm (Fig. 5). Follow-up CT examinations were performed every 6 months following surgery, and the patient was alive 2 years after the second surgery. In August 2008, the patient again presented with symptoms of dysphagia and a giant tumor was detected in the posterior mediastinum by

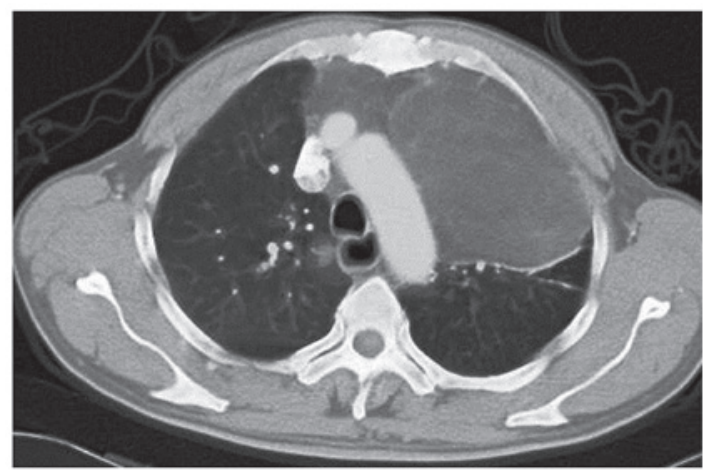

Figure 4. Chest computed tomography indicating a large mass in the anterior mediastinum and left cavity.

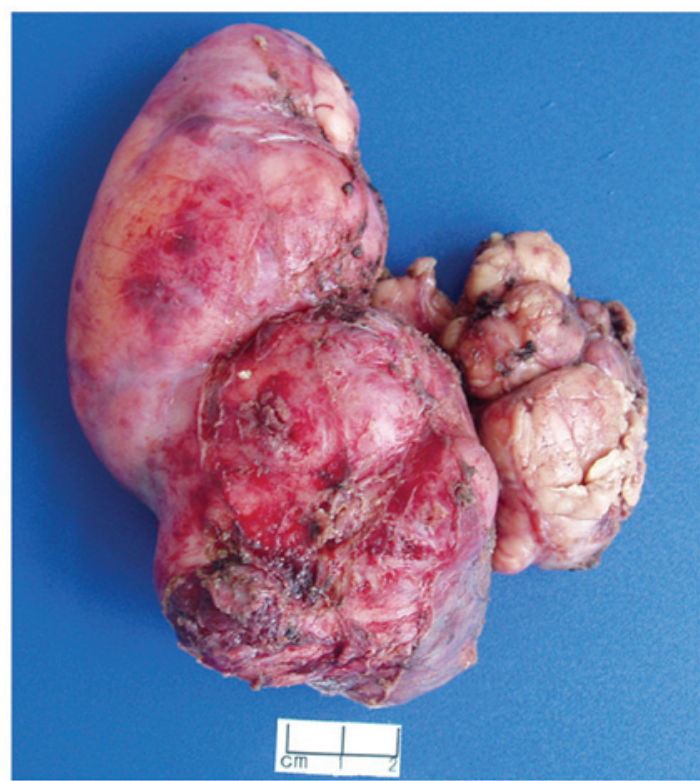

Figure 5. Macroscopic view of the tumor. The tumor weighed $980 \mathrm{~g}$ and its dimensions were $16 \times 12 \times 8 \mathrm{~cm}$.

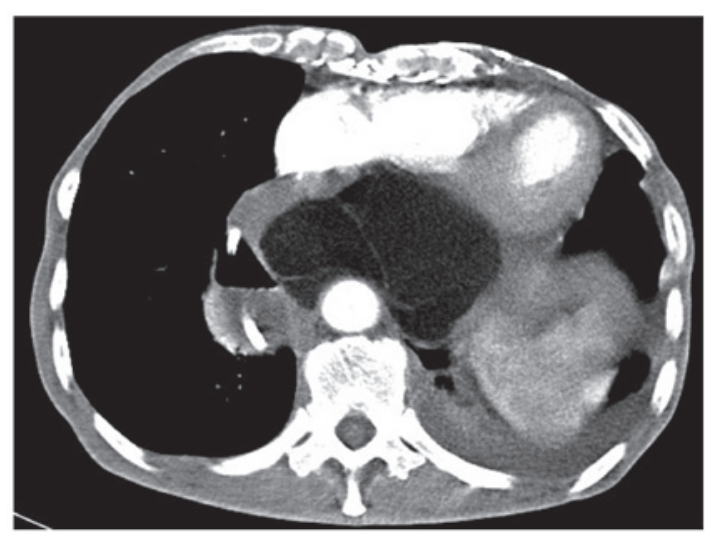

Figure 6 . Chest computed tomography indicating a large mass in the posterior mediastinum.

chest CT (Fig. 6). However, the patient refused any treatment and succumbed after 3 months.

Written informed consent was obtained from the family of the patient for the publication of the present study. 


\section{Discussion}

Liposarcoma is a mesenchymal tumor that is commonly derived from adipocytes (5). It is one of the most frequent malignant soft tissue tumors in adults (5). However, primary liposarcomas of the mediastinum are extremely rare. Thus far, $<130$ cases have been reported in the published literature (3). According to statistics reported by Kashu et al (3), the most common sites of these tumors are the lower extremities (75\%) and the retroperitoneum (20\%). In the early stage primary mediastinal liposarcomas, patients do not typically exhibit any obvious clinical symptoms. However, as in the present case, patients present with various different symptoms as the tumor grows, depending on its location and on the compression of neighboring structures, such as progressive dysphagia, dyspnea, chest pain, shortness of breath or hoarseness.

Over several years, the common characteristics of mediastinal liposarcoma have been increasingly recognized. It is impossible to be certain whether the mass is benign or malignant by performing imaging examinations alone, such as chest $\mathrm{X}$-ray, CT and MRI, and histopathology is used for a definitive diagnosis. However, they do assist in diagnosing mediastinal liposarcoma; on MRI, T1-weighted images reveal the fatty tissue with high signal intensity, whereas in T2-weighted images, the signal intensity diminishes (6).

Due to of the great variety of histological presentations, primary mediastinal liposarcoma may be easily misdiagnosed. Therefore, the differential diagnosis of mediastinal liposarcomas should include lipoma, teratoma, mesothelioma, fibrosarcoma, leiomyosarcoma and neurogenic tumor.

The most effective treatment for primary mediastinal liposarcoma is surgery. The efficacy of this therapeutic method depends on the extent of resection, with total resection performed when possible. According to the published literature, aggressive surgical intervention appears to prolong survival and favor good quality of life (7). For example, Kashu et al (3) reported a case of large mediastinal liposarcoma that was successfully treated with radical resection. The patient remained alive with no evidence of disease recurrence at 22 months after the surgery. Furthermore, Decker et al (4) reported a case of large mediastinal liposarcoma occupying the majority of the right chest, and abutting the great vessels and pericardium. Similar to the present study, a thoracoscopic approach was used for exploration and surgical excision of this large mediastinal mass. The patient remained disease-free for almost 3 years after the resection (8). A few cases also report the use of radiotherapy, chemotherapy or other methods for the treatment of primary mediastinal liposarcomas (7); for example, Shoji et al (9) reported the case of a patient with mediastinal liposarcoma treated by radiofrequency ablation (RFA). The patient was followed up for an additional 12 months without any other treatment, and CT revealed that the lesion had not progressed in size. Therefore, RFA has been demonstrated to be an effective option for patients who are not well suited to undergo a further surgical resection (9). A number of authors consider radiotherapy to be useful in the adjuvant treatment of unresectable tumors; however, the role of adjuvant chemotherapy remains controversial (10).

In conclusion, the current study presents a rare case of liposarcoma arising from the mediastinum in a 63-year-old man. Tumor resection was successfully performed, however, at a follow-up examination 12 mouths after surgery, recurrence was identified in the posterior mediastinum. Subsequently, the tumor was successfully removed. However, recurrence again occurred in the posterior mediastinum 2 years later. The patient refused further treatment and succumbed after 3 months. A diagnosis of mediastinal liposarcoma was made based on the clinical features, imaging findings and pathological findings. The current case and previous studies indicate that careful long-term follow-up with radiological imaging is necessary to rule out recurrence or metastasis.

\section{References}

1. Punpale A, Pramesh CS, Jambhekar N and Mistry RC: Giant mediastinal liposarcoma: A case report. Ann Thorac Cardiovasc Surg 12: 425-427, 2006

2. Wick MR: The mediastinum. In: Diagnostic Surgical Pathology. Sternberg SS and Antonioli DA (eds). 3rd edition. Vol 1 and 2. Lippincott Williams and Wilkins, Philadelphia, PA, pp1147-1208, 1999.

3. Kashu Y, Yukumi S, Tsunooka N, Tanigawa K, Arakane M, Nakagawa $\mathrm{H}$ and Kawachi K: Successful resection of a massive mediastinal liposarcoma that rapidly extended into the entire left thoracic cavity: Report of a case. Surg Today 42: 68-71,2012.

4. Decker JR, de Hoyos AL and Decamp MM: Successful thoracoscopic resection of a large mediastinal liposarcoma. Ann Thorac Surg 92: 1499-1501, 2011.

5. Teschner M and Lüllig H: Diagnosis and treatment of primary mediastinal liposarcoma. Pneumologie 57: 22-26, 2003 (In German).

6. Munk PL, Lee MJ, Janzen DL, Connell DG, Logan PM, Poon PY and Bainbridge TC: Lipoma and liposarcoma: Evaluation using CT and MR imaging. AJR Am J Roentgenol 169: 589-594, 1997.

7. Gasiorowski L, Dyszkiewicz W and Piwkowski CT: An unusual case of giant primary mediastinal liposarcoma. Thorac Cardiovasc Surg 57: 247-248, 2009.

8. Meyer M, Holzhausen HJ, Neef H and Zerkowski HR: Primary liposarcomas of the mediastinum. Langenbecks Arch Chir Suppl Kongressbd 115: 369-373, 1998 (In German).

9. Shoji F, Taketomi A, Yano T and Maehara Y: Intraoperative radiofrequency ablation in an open thoracotomy setting for the new treatment of mediastinal liposarcoma: Report of a case. Surg Today 41: 992-994, 2011.

10. Saad R Jr, Dorgan Neto V, Gonçalves R, Botter M and Siqueira LC: Mediastinal liposarcoma: a case report. J Bras Pneumol 34: 55-58, 2008 (In Portuguese). 